\title{
Proton Synchrotron Radiation Diagnostics at HERA
}

\author{
G. Kube, G. Priebe, Ch. Wiebers, and K. Wittenburg \\ Deutsches Elektronen-Synchrotron DESY, Notkestrasse 85, D-22607 Hamburg, Germany
}

\begin{abstract}
At HERA a proton synchrotron radiation beam emittance monitor has been installed which utilizes the radiation produced in the fringe field of a vertical deflecting dipole magnet. The beam spot is imaged onto the chip of a standard CCD camera by means of a concave mirror. An interference filter together with a polarization filter serve for resolution improvements. While the monitor was used for the first time in standard operational mode during the previous HERA run period, the set-up was improved in the last shut-down. With start of the new run period the monitor is in a stage of re-comissioning and the first results are presented.
\end{abstract}

Keywords: Emittance diagnostics, Synchrotron radiation, Edge Radiation, Proton beam. PACS: 07.85.Qe, 41.60.Ap, 41.85.Ew

\section{INTRODUCTION}

The "Hadron Elektron Ring Anlage" HERA at DESY provides collisions between a $920 \mathrm{GeV}$ proton and a $27.5 \mathrm{GeV}$ electron beam. In order to understand the luminosity delivered to the experiments a precise online control of both beam emittances is highly desirable. Due to its non-destructive nature optical synchrotron radiation (SR) originating from the center of dipole magnets is a versatile tool for beam profile measurements and is used in a large number of circular electron accelerators. In proton accelerators an additional problem arises due the larger particle rest mass $m_{0} c^{2}$ : the critical frequency $\omega_{\mathrm{c}}=3 \mathrm{c} \gamma^{3} /(2 \rho)$ characterizing the SR emission spectrum with $\gamma=\mathrm{E} / \mathrm{m}_{0} \mathrm{c}^{2}$ the Lorentz factor and $\rho$ the magnet bending radius is shifted towards the infrared or even lower. Optical elements are not easily available in these spectral regions and the image resolution is deteriorated due to an increased contribution from diffraction broadening. In order to overcome these limitations the SR emission spectrum can be extended to higher frequencies if radiation produced in dipole fringe fields or in short magnets is used which contains sufficient intensity to render possible beam profile measurements.

The first profile monitor based on this principle was realized at the SPS [1]. The fringe field between two successive dipole magnets was used for radiation generation. While this monitor worked only at energies above about $270 \mathrm{GeV}$ it was replaced later by an undulator which extended the usable energy range [2]. A similar beam profile monitor has been installed at the Tevatron where synchrotron light produced from protons (antiprotons) at the upstream edge of a superconducting magnet is observed [3]. For the LHC it is also planned to use this type of monitor for transverse beam diagnostics. In order to optimize the performance over the whole energy range from 
$450 \mathrm{GeV}$ up to $7 \mathrm{TeV}$, a superconducting undulator together with a separation dipole will act as radiation source $[4,5]$.

At HERA a proton synchrotron radiation beam profile monitor has been installed which utilizes the radiation produced in the fringe field of a vertical deflecting dipole magnet. The beam spot is imaged onto the chip of a standard CCD camera by means of a concave mirror. An interference filter together with a polarization filter serve for resolution improvements. The monitor was used for the first time in standard operational mode during the previous HERA run period. After a setup improvement during the last shut-down the monitor is now in a stage of re-comissioning.

In the subsequent paragraph a brief overview of the basic radiation properties will be given before the monitor setup together with the first results are presented.

\section{THEORY OF PROTON EDGE RADIATION}

In the following the basic properties of proton SR generation in bending magnet fringe fields will be outlined. An illustrative explanation of the "frequency boost" is given based on the SR frequency spectrum. For SR based profile measurements the knowledge of resolution broadening contributions is essential. Therefore the spectral angular distribution is discussed and the calculation of resolution broadening is summarized, taking into account radiation propagation through the monitor's optical system.

\section{Frequency Spectrum}

In order to understand the intensity increase at higher frequencies in fringe fields it is helpful to recall the SR radiation field properties in the time domain, see for example Ref. [6]. Both quantities are simply related by the square of the absolute value of the Fourier transform, c.f. Eq.(1).

$$
\frac{d^{2} P}{d \Omega d \omega} \propto\left|\vec{E}_{\omega}\right|^{2} \quad \text { with } \quad \vec{E}_{\omega}=\frac{1}{\sqrt{2 \pi}} \int_{-\infty}^{+\infty} d t \vec{E}(t) \exp \{i \omega t\} .
$$

Fig.1(a) shows a simple illustration of the SR typical frequency as given in various textbooks. The typical frequency is determined by the length of the radiation pulse seen by an observer. Due to the small SR emission angle of about $\theta \sim 1 / \gamma$ only radiation from a small orbit interval between point $\mathrm{A}$ and $\mathrm{B}$ can be observed. The length of the radiation pulse is nothing other than the difference in travel time $\Delta t=t_{e}-$ $\mathrm{t}_{\gamma}$ between the electron and the emitted SR photon going from point A to B. In Fig. 1(b) the time dependence of the radiation field's horizontal (sigma) polarization component $\mathrm{E}_{\sigma}$ is plotted as seen by the observer ${ }^{1}$, assuming the radiation is originating from the center of an (infinitely) long dipole magnet. For an observation point located in the orbit plane the zeros of $E_{\sigma}$ are determined by $1 / \omega_{\mathrm{c}}$, i.e. the reciprocal of the SR critical frequency. In order to increase the intensity at higher photon energies the Fourier transform Eq.(1) of the smooth radiation field $\mathrm{E}_{\sigma}$ in Fig. 1(b) must contain higher frequencies. This can be achieved in the time domain by a sharp cut-off of the

\footnotetext{
${ }^{1}$ The vertical (pi) polarization component is not considered because its intensity contribution is typically much lower.
} 
(a)

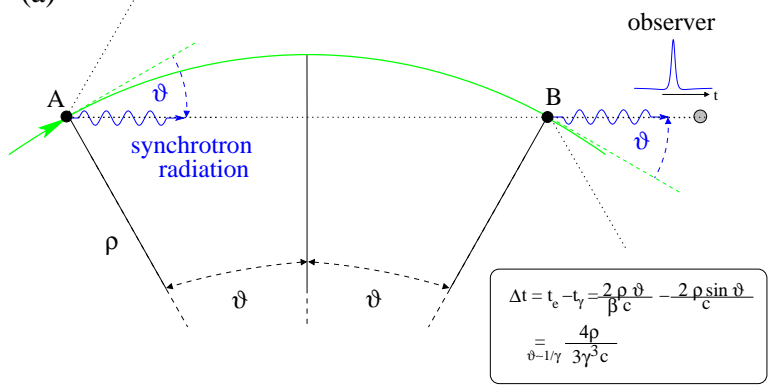

(b)

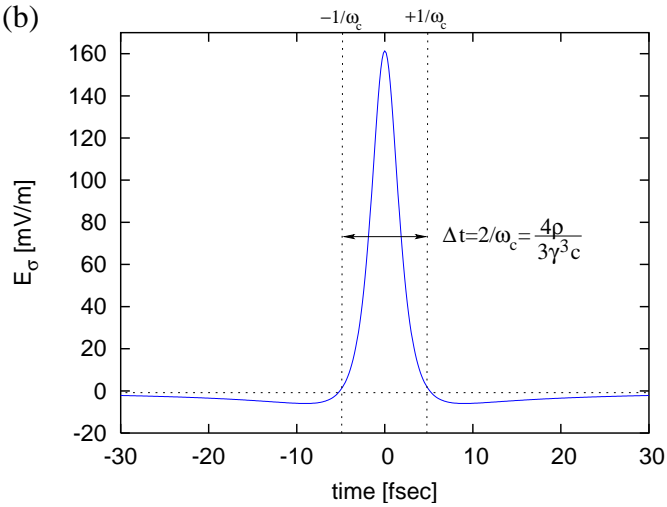

FIGURE 1. (a) Geometry for the derivation of the SR time structure. (b) Time dependence of the horizontally polarized radiation field $\mathrm{E}_{\sigma}$ as calculated for a $920 \mathrm{GeV}$ proton in an (infinitely) long bending magnet with bending radius $\rho=2163 \mathrm{~m}$. The observation point is in the orbit plane.

radiation field. This is the case if radiation from a magnet edge is observed instead from the central region. The influence of this effect is illustrated in Fig. 2 for the HERA proton beam at $920 \mathrm{GeV}$. In the top the radiation fields in the time domain are plotted for the central and fringe fields of a bending magnet with $\rho=2163 \mathrm{~m}$, assuming a hard edge model. Below the radiated power is shown calculated by Fourier ransforming the radiation field according to Eq.(1). As can be seen in Fig. 2 the spectral content of edge radiation contains orders of magnitude more intensity than
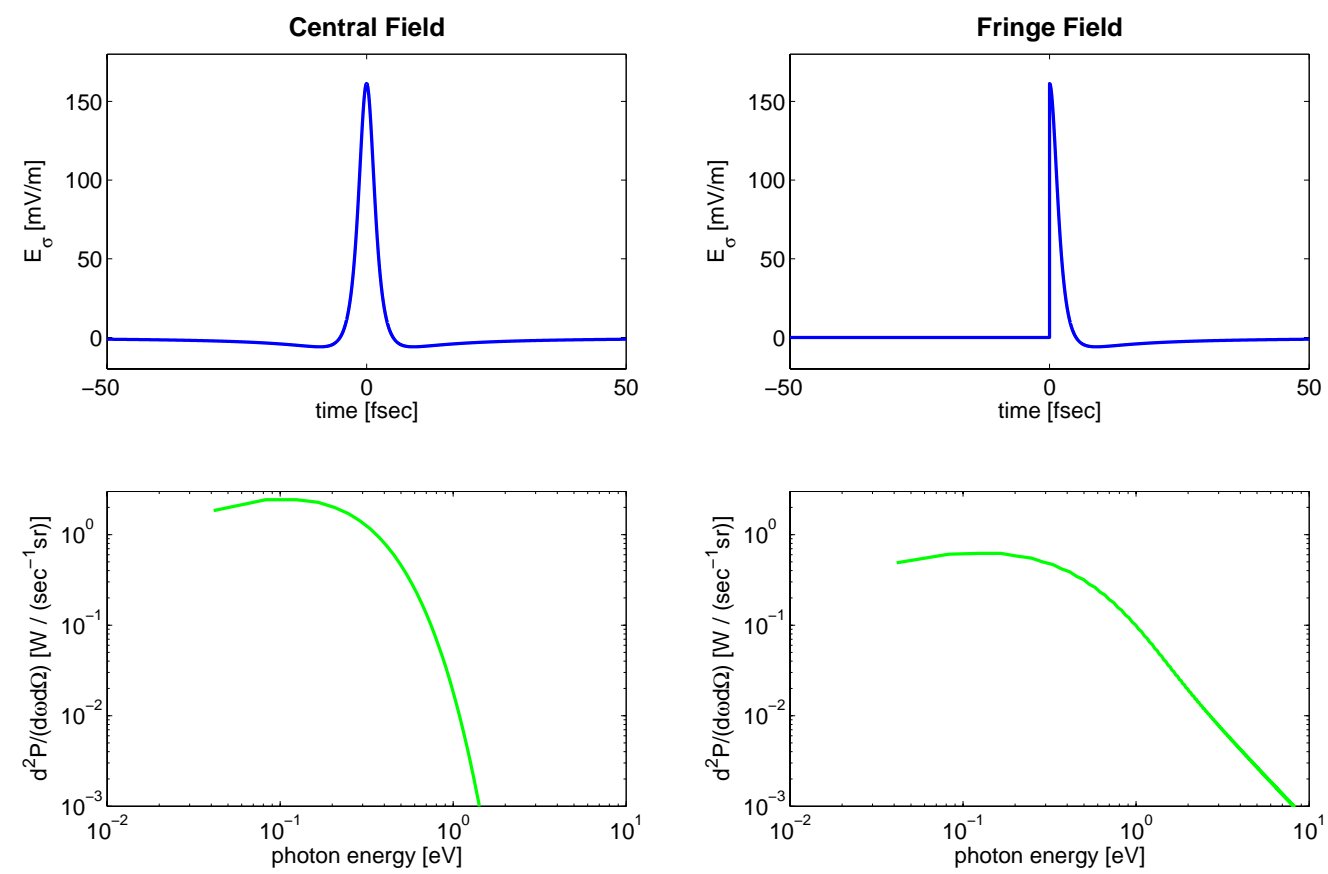

FIGURE 2. Top: Time dependence $\mathrm{E}_{\sigma}$ for (left) radiation from the central part and (right) radiation from the upstream edge of a bending magnet. The calculations are based on the parameters of the HERA monitor. Bottom: spectral power density calculated by Fourier transforming the radiation field according to Eq.(1). 
$\mathrm{t}$ radiation from the central field at photon energies above $1 \mathrm{eV}$, thus render it possible to perform beam diagnostics in the optical spectral region.

In a similar way short magnet radiation can be understood. In this case the radiation field in the time domain is truncated by upstream and downstream magnet edges symmetrically around $t=0$. In view of high frequency components a reasonable magnet length corresponds to a cut-off time $t_{c}$ which is smaller than $\left|1 / \omega_{c}\right|$, cf. Fig. 1(b). In this case the length of the radiation pulse is determined by the magnet length rather than by the SR characteristic angle $\vartheta \sim 1 / \gamma$, c.f. Fig. 1(a).

\section{Spectral Angular Distribution And Resolution Broadening}

A rigorous treatment of the angular-spectral distribution and polarization of SR from short magnets and special edge profiles of magnets can be found in Ref. [7]. For the special case that the edge profile is parameterized using an error function, i.e.

$$
B(z)=\frac{B_{0}}{2}(1+\operatorname{erf}(z / L))
$$

with $L$ the magnetic length, the number of photons per solid angle and frequency interval reads ${ }^{2}$

$$
\frac{d^{2} N}{d \Omega d(\hbar \omega)}=\frac{8 \alpha}{\pi^{2}} \mu_{N}^{2} \frac{\gamma^{6}}{(\hbar \omega)^{3}} B_{0}^{2} f^{2} \exp \left[-2\left\{\frac{L\left(1+\vartheta^{2} \gamma^{2}\right)}{4 \gamma^{2} \hbar c} \hbar \omega\right\}^{2}\right] .
$$

Here $\alpha$ is the fine structure constant, $\mu_{\mathrm{N}}$ the nuclear magneton (in the case of protons) and $f$ is a factor describing the polarization state which is given by

$$
f^{2}=f_{x}^{2}+f_{y}^{2} \quad \text { with } \quad f_{x}=\frac{\gamma^{2}\left(\vartheta_{x}^{2}+\vartheta_{y}^{2}\right)-1}{\left(1+\vartheta^{2} \gamma^{2}\right)^{3}}, \quad f_{y}=\frac{2 \gamma^{2} \vartheta_{x} \vartheta_{y}}{\left(1+\vartheta^{2} \gamma^{2}\right)^{3}} \text {. }
$$

The observation geometry is chosen such that the $\mathrm{x}$ axis is perpendicular to the particle beam direction $(z)$ in the orbit plane and forms a Cartesian system together with the y axis, i.e. $f_{x}=0$ corresponds to the case of $\pi$ polarization and $f_{y}=0$ to $\sigma$ polarization. With $(x, y)$ the coordinates in the observation plane and $R$ the distance between source point and observation plane the observation angles are $\vartheta_{\mathrm{x}}=x / R, \vartheta_{\mathrm{y}}=$ $y / R$.

In Fig. 3 calculated spectral angular distributions are shown according to Eq.(3). The $\sigma$ component has a shape similar to SR from the central part of a bending magnet with a maximum on axis. In contrast to that the $\pi$ component has fourfold symmetry instead of a twofold one while the minimum on axis remains. Compared to the intensity of the $\sigma$ polarization component the $\pi$ component is less by more than one order of magnitude. Nevertheless for purposes of beam diagnostics the contribution of the latter polarization component should be avoided because the broader angular distribution leads to an increase of diffraction broadening, thus spoiling the spatial resolution of a beam profile monitor.

Unfortunately the real fringe field of the HERA bending magnets shown in the insert of Fig. 5 cannot be parameterized in a simple form by an analytical expression

\footnotetext{
${ }^{2}$ In the formula of Ref. [7] the factor 2 is missing in the exponential.
} 

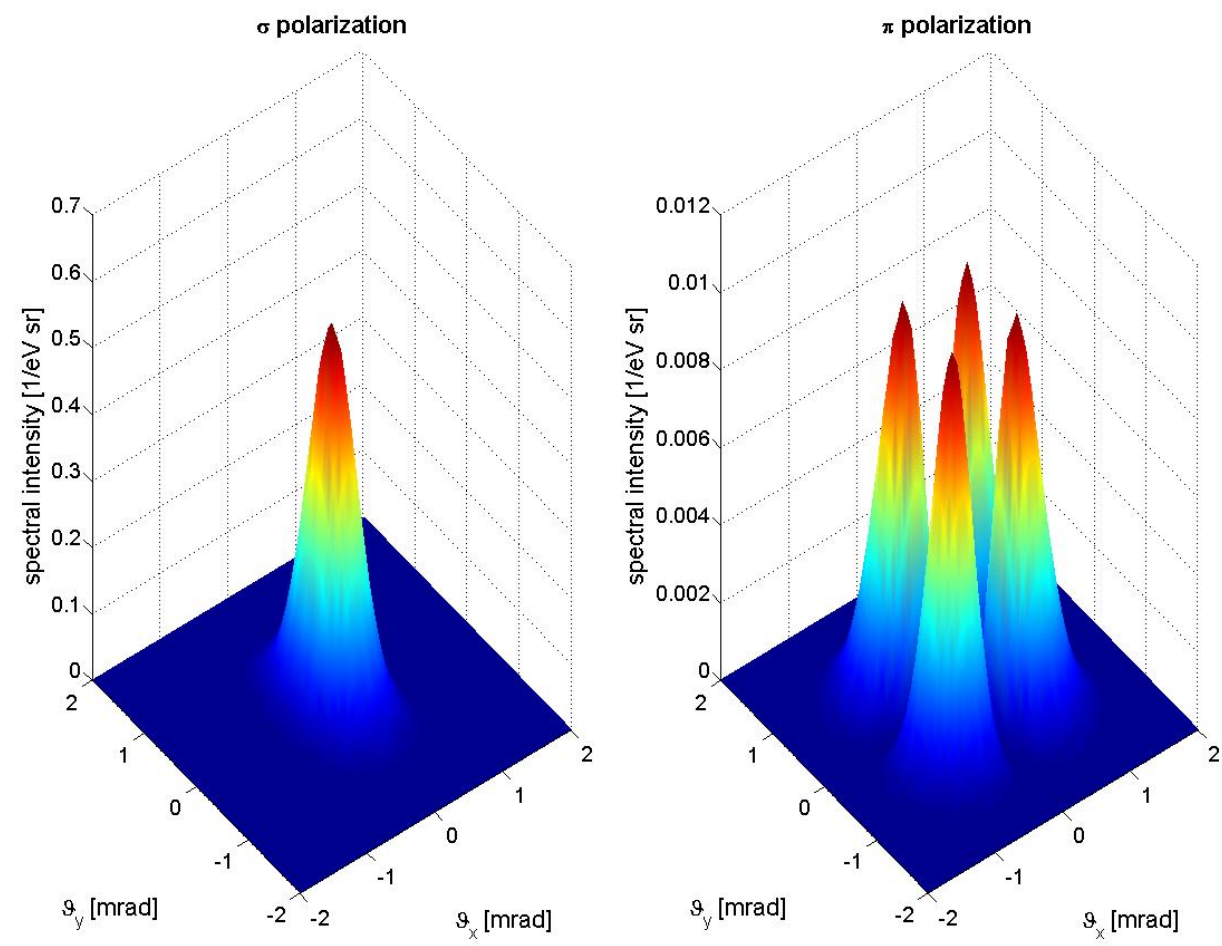

FIGURE 3. Spectral angular distribution, calculated for the HERA monitor according to Eq.(3): beam energy $\mathrm{E}=920 \mathrm{GeV}$, wavelength of observation $\lambda=500 \mathrm{~nm}$ (corresponding to a photon energy of 2.5 $\mathrm{eV}$ ), fringe field length $\mathrm{L}=9.2 \mathrm{~cm}$, and magnet strength $\mathrm{B}_{0}=1.42 \mathrm{~T}$.

as given in Eq.(2). Therefore in order to describe the resolution broadening effects in both planes calculations have been performed with the computer code SRW [8]. With this code, the initial SR electric field is computed in the frequency domain using a high-accuracy near-field method. The method is based on the Fourier transform of the retarded potentials which are used to derive an expression for the electric field [9] and it is exact in the sense that the velocity field term is not neglected as it is in the case for calculations based on the Fourier transform of the Liénard-Wiechert fields. The computation preserves all the phase terms that are necessary for further propagation of the radiation through optical components. SR propagation is implemented in the frame of scalar diffraction theory applying the methods of Fourier optics. For purposes of beam diagnostics, this method of calculating SR fields has the advantage that resolution broadening contributions like diffraction and depth of field are directly included.

For the calculation of the resolution broadening contributions the HERA SR monitor was modeled in a simple way: the influence of the extraction mirror in both planes was described by a rectangular aperture with the corresponding mirror dimensions. Finally the beam profile image was calculated at the position of the CCD camera after propagation through the optical system for which a simple achromat in thin lens approximation was assumed. 


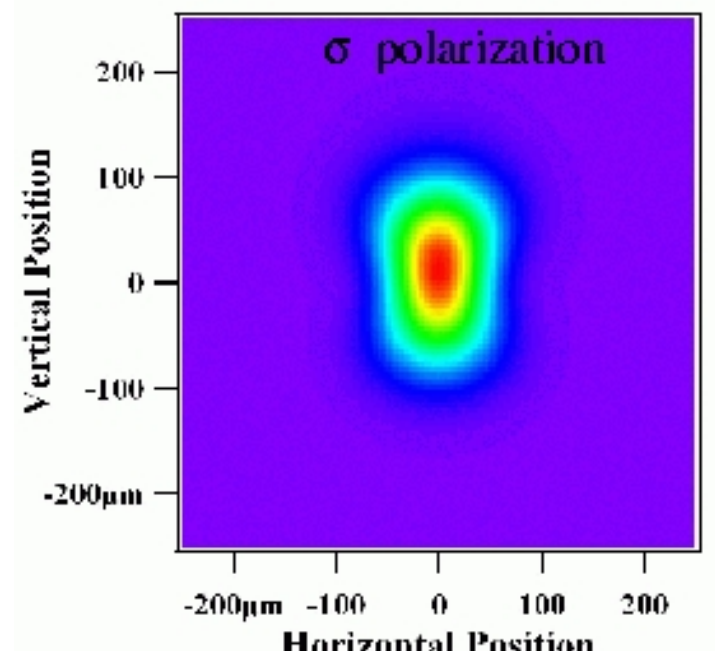

Horizontal P'osition

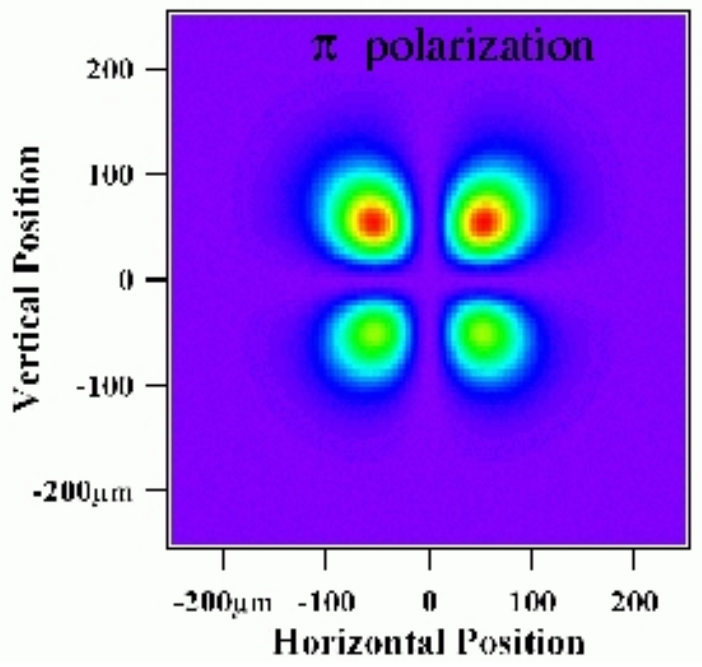

Horizontal l'osition

FIGURE 4. Calculated images of filament beam profiles after propagation through the optical system. Parameters of the calculation are the same as those used in Fig.3.

Fig. 4 shows the calculated images of a filament beam profile for both polarization states. For the determination of resolution broadening contributions only the $\sigma$ polarization component is considered. The filament beam image was fitted and the resulting image size was transformed back in the source plane using the geometrical magnification of the optical system. The resolution broadening contributions so determined are

$$
\Delta \sigma_{x} \approx 84 \mu \mathrm{m}, \quad \Delta \sigma_{y} \approx 144 \mu \mathrm{m} .
$$

\section{MONITOR SETUP}

In luminosity operation the HERA proton beam energy is $\mathrm{E}=920 \mathrm{GeV}$. According to the beam optical design parameters, in this mode the beam should have a horizontal $(1 \sigma)$ size of $\sigma_{\mathrm{x}}=1611 \mu \mathrm{m}$ and a vertical size of $\sigma_{\mathrm{y}}=454 \mu \mathrm{m}$ at the location of the radiation source point. Therefore the particle beam size is much larger than the expected resolution broadening contributions which are nearly negligible at least for the analysis of the horizontal beam profile.

In Fig. 5 a sketch of the monitor setup is shown. After passage through the HERMES experiment the proton beam is separated in the vertical direction from the electron beam by a triplet of normal conducting bending magnets, each deflecting about 1.9 mrad. Radiation from the upstream edge of the first bending magnet is used for image formation.

The task of the optical system is to provide an image of the beam onto the chip of the CCD camera. For this purpose a reflecting telescope of Herschelian type was built. The emitted SR radiation is extracted out of the vacuum system by a flat surface mirror (M1) through a glass window without disturbing the proton beam. The mirror is moveable vertically and is located $15.27 \mathrm{~m}$ away from the upstream magnet yoke edge of the first bending magnet. Fig. 6(a) shows a photograph of the extraction mirror. A 
second flat surface mirror (M2) which is rotateable and $0.73 \mathrm{~m}$ away from the first one reflects the extracted radiation onto a spherical mirror which serves as the imaging element. The distance between the mirrors is $0.74 \mathrm{~m}$. The spherical mirror has a focal length of $\mathrm{f}=4.72 \mathrm{~m}$, a diameter of $90 \mathrm{~mm}$ and is rotateable around two axes. In Fig. 6(b) a photograph of the spherical mirror setup is shown. The overall magnification of the optical system is about 0.4 .

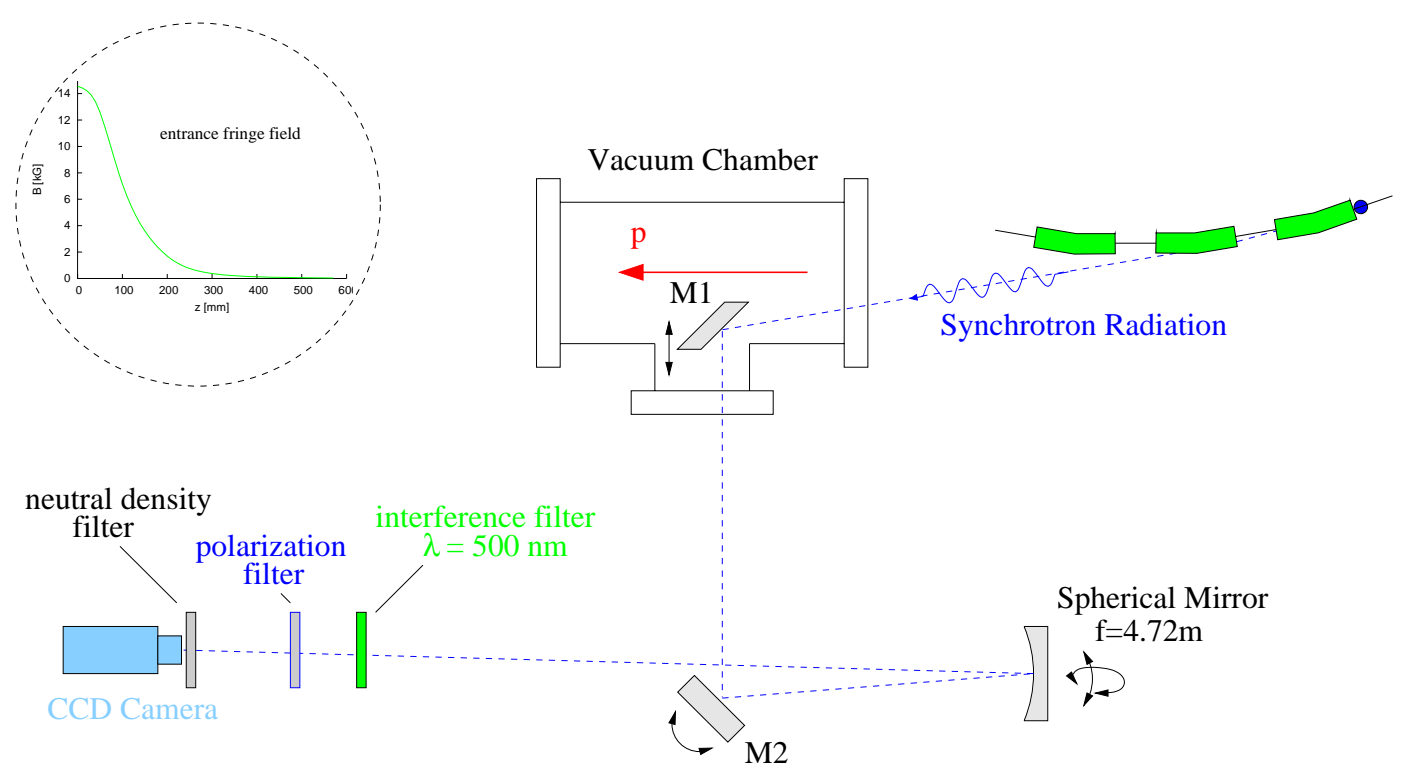

FIGURE 5. Schematic picture of the profile monitor setup. The insert shows the measured shape of the bending magnet fringe fields.

The beam spot is imaged onto the chip of a standard CCD camera (JAI CV-M300E with $768 \times 494$ pixels of size $11.6 \mu \mathrm{m} \times 13.5 \mu \mathrm{m}$ ) which should be $6.57 \mathrm{~m}$ away from the spherical mirror for real imaging. The video output of the camera is fed to a commercially available 8 bit PCI framegrabber board (Data Translation DT3155) for digitization and finally analyzed by a standard personal computer.

In order to minimize chromatic errors and to improve the diffraction limited resolution an interference filter (central wavelength $500 \mathrm{~nm}$, FWHM $35 \mathrm{~nm}$ ) is used together with a polarization filter. Neutral density filters serve to adjust the incoming light intensity to avoid saturation of the camera chip.

In the first stage of the monitor setup the camera was mounted at a fixed position (setup 1). However, based on the preceding calculations it can be demonstrated that the contribution of the resolution broadening effects strongly depends on the distance between camera and spherical mirror, i.e. slight differences of the distance from the design lead to a strong deterioration of the beam profile. In addition the neutral density filters as well as the polarization filters could not be interchanged remotely. Therefore it was decided to improve the detector part of the monitor during the last HERA shut down period. In the beginning of this year the old camera setup was replaced by a new detector chamber (setup 2) which is shown in Fig. 6(c). With this setup it is possible to adjust the distance between mirror and camera as well as to change polarization 

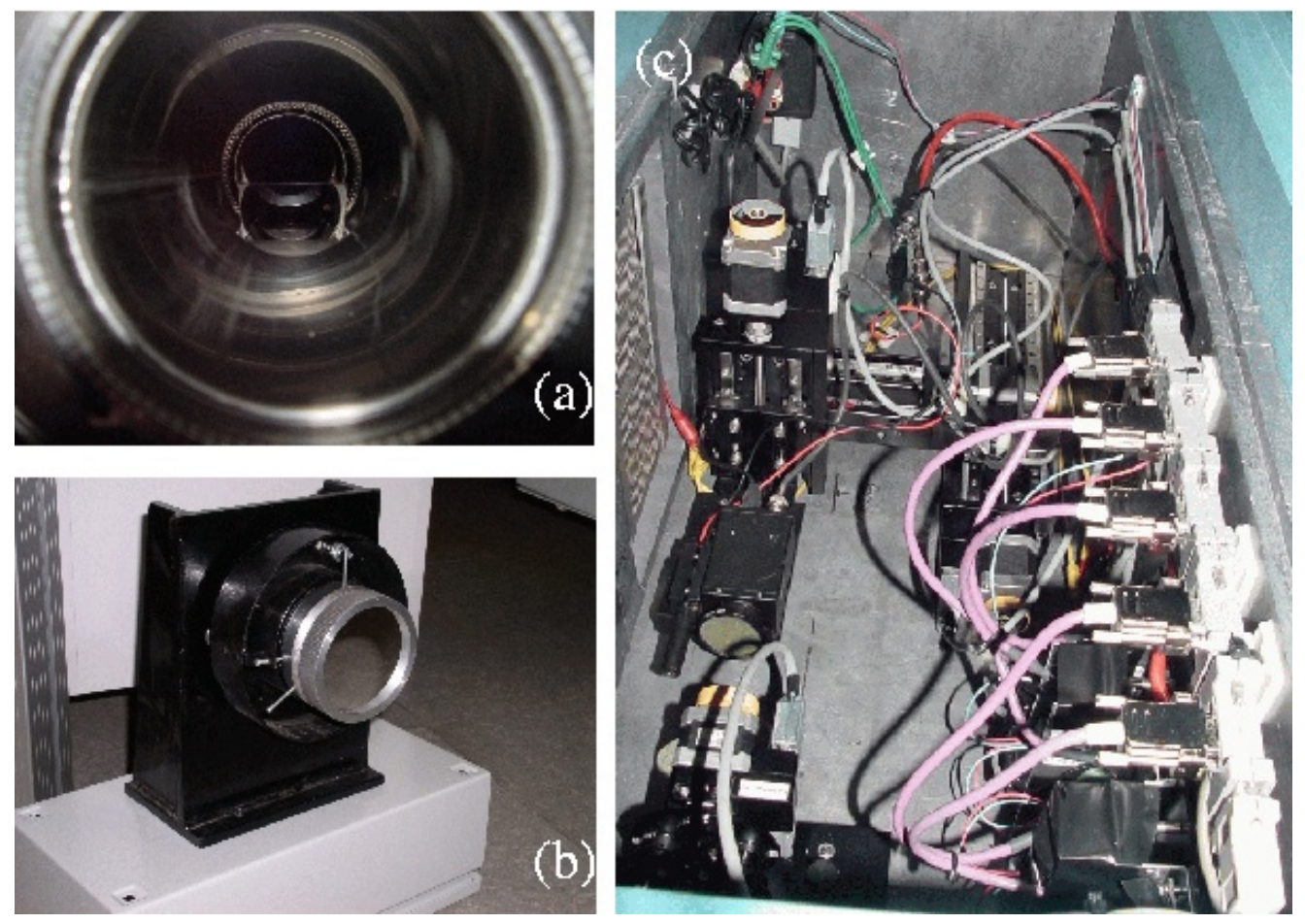

FIGURE 6. Photos of the monitor setup. (a) View in the beam tube on the extraction mirror. (b) Setup of the spherical mirror. (c) The new detector setup. The camera together with a photomultiplier are mounted on an alignment system which consists of three stepper motors used to adjust the detectors in three translational degrees of freedom. Two filter wheels in front of the detectors allow to attenuate the intensity and to select the polarization direction.

direction and neutral density filters remotely controlled. In addition a photomultiplier was added which should help to align the monitor in the commissioning stage. For background shielding against neutrons the whole detector setup is installed in a box of Tekalen which is a material with high hydrogen concentration and doped with $16.1 \%$ boron oxide. In addition the box is faced with a lead shielding against gamma background. With the start of the new HERA run period the monitor is in a stage of recomissioning.

\section{RESULTS}

During the last HERA run period the monitor was used in the standard operational mode for the first time with setup 1. Fig. 7 shows a monitor screen shot taken during this time. The measured beam profile is fitted with a two-dimensional normal distribution and the emittances are evaluated based on the theoretical beam optics. The results of the online analysis are included in the HERA archive with an update rate of about $10 \mathrm{~Hz}$ and are available for further offline analyses.

As can be seen from Fig. 7 the horizontal beam profile is well reproduced. However, in the vertical direction the profile is slightly distorted. The reason is probably a reflection of the incoming light at the entrance of the detector chamber which should be eliminated in the new setup 2 . 


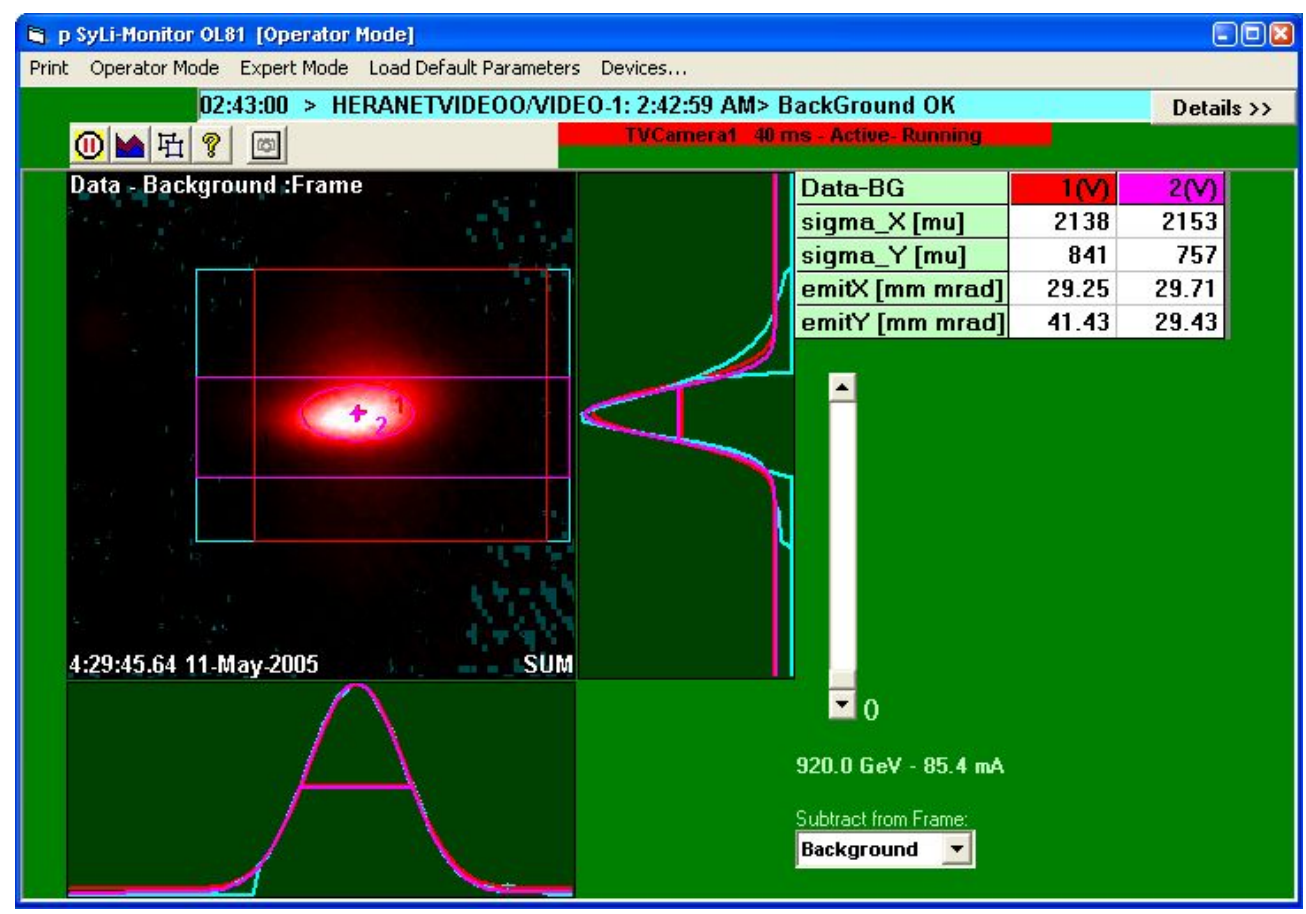

FIGURE 7. Screen shot of the HERA synchrotron radiation emittance monitor. The transverse proton beam profile is continuously observed and analyzed.

While the evaluation of the vertical emittance is questionable due to the influence of the reflection, the horizontal emittance deduced from the measured profile is reasonable. A comparison between measurements from a wire scanner and the proton SR monitor show that the emittances from the latter are larger. The reason is presumably that the distance between spherical mirror and camera was not correct, a problem which should be overcome in the new setup 2 by online adjustment.
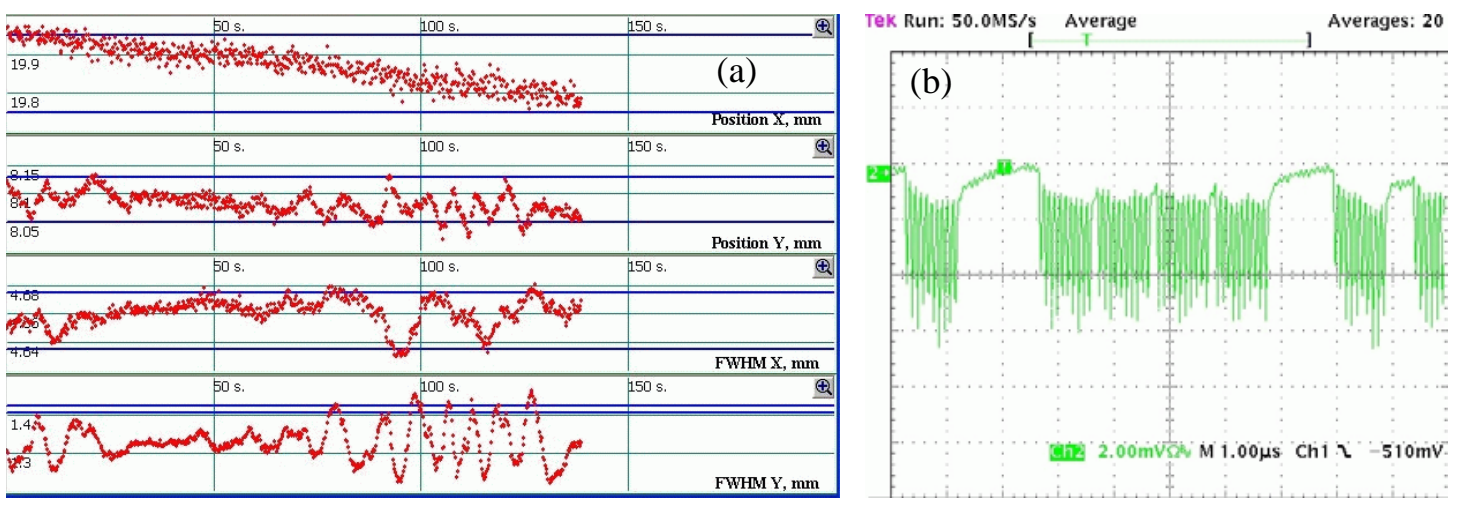

FIGURE 8. (a) Screen shot of the HERA synchrotron radiation emittance monitor. The time evaluation of the horizontal (x) and vertical (y) beam position is shown together with the fitted beam sizes (FWHM) for the last $200 \mathrm{~s}$. (b) Photomultiplier signal, observed with an oscilloscope. The HERA proton fill pattern is clearly recognized (from the total of 150 bunches only a fraction is represented). 
Apart from the determination of the absolute emittance the monitor is a versatile tool for the study of dynamical beam effects, for which one needs to measure relative changes in the transverse beam profile. In Fig. 8(a) an example of such a measurement is shown. In this case the proton collimators in the HERA ring were moved towards the beam which excited the beam to collective oscillations.

After the improvements and the start up of HERA the monitor is now in a process of re-comissioning. As can be seen in Fig. 8(b) proton SR is already detected by the photomultiplier. The multiplier signals observed with an oscilloscope reproduce nicely the fill pattern of the HERA proton ring. However, the alignment procedure of the imaged beam spot onto the chip of the CCD camera through the optical telescope is still in process, and the monitor should be back soon in standard operation.

\section{SUMMARY}

This article summarizes the status of the HERA proton synchrotron radiation monitor for online transverse profile measurements. The basic properties of SR generation in fringe fields of a bending magnet were described and it was shown that imaging with edge radiation can be managed with good resolution in the optical spectral region. After a description of the monitor setup first results were presented. While the deduced horizontal beam emittance is slightly larger compared to wire scanner measurements the monitor serves as a versatile tool for the study of dynamical beam effects. After improvement of the setup during the last HERA shut down the monitor is now in a stage of re-comissioning.

\section{ACKNOWLEDGMENTS}

The authors would like to thank A. Affeldt, M. Borchardt, R. Fischer and R. Zahn for their valuable contributions during the design and construction phase, O. Grimm for stimulating discussions concerning SR properties in the time domain, and $\mathrm{M}$. Lomperski for carefully reading the manuscript.

\section{REFERENCES}

1. R.Bossart, J. Bosser, L. Burnod, R. Coisson, E. D’Amico, A. Hofmann, and J. Mann, Nucl. Instr. and Meth. 164 (1979), pp. 375-380.

2. J. Bosser, L. Burnod, R. Coisson, G. Ferioli, J. Mann, and F. Méot, "Characteristics of the radiation emitted by protons and antiprotons in an undulator", CERN-SPS/83-5 (1983).

3. A.A. Hahn and P. Hurh, "Results from a prototype beam monitor in the Tevatron using synchrotron light", in 1992 IEEE Particle Accelerator Conference 1991, pp.1177-1179.

4. F. Méot, L. Ponce, J. Bosser, R. Jung, "Diagnostics with synchrotron radiation of the LHC proton beams", in Proceedings of the European Particle Accelerator Conference, Paris, France, 2002, pp.1945-1947.

5. L. Ponce, R. Jung, F. Méot, "LHC proton beam diagnostics using synchrotron radiation", CERN-2004-007 (2004).

6. A. Hofmann, "The Physics of Synchrotron Radiation", Cambridge Monographs on Particle Physics, Nuclear Physics and Cosmology 20 (2004)

7. R. Coisson, Phys. Rev. A20 (1979), pp.524-528

8. O. Chubar and P. Elleaume, Proceedings of the European Particle Accelerator Conference, Stockholm, Sweden, 1998, pp.1177-1179

9. O. Chubar, Rev. Sci. Instrum. 66 (1995) 1872 